\title{
4 Winnenden: Erleben einer zeitlichen Zäsur
}

Schon seit dem Erfurter Ereignis am 26.4.2002 ist Schul-Amok für jedermann in Deutschland als Realität präsent. Schulen, Polizei und Politik beschäftigen sich seitdem verstärkt mit dem neuen Phänomen Schul-Amok und School Shooting. In den Schulen scheint seitdem das Grundvertrauen erschüttert zu sein, vor allem weil nach jedem Ereignis Nachahmer und Trittbrettfahrer Schulen und Polizei in Atem halten. Unter Handlungszwang geratene Politiker neigen dann zu populistischen Maßnahmen und kurzfristigem Agieren und die Politik versucht jedes Mal die Situation mit der Verschärfung des bisherigen Waffengesetzes, mit der Einführung einer neuen Sicherheitstechnik und mit Hilfe von neuen Notfallplänen in den Schulen zu beruhigen. Viele Menschen fragen sich beunruhigt, ob der nächste Amoklauf an einer Schule nur noch eine Frage der Zeit ist.

Immer wieder tauchten nach dem ersten Amoklauf in Erfurt reflexartig gestellte Fragen auf:

- Was hat man im Vorfeld dieser Taten übersehen oder versäumt?

- Hauschild (2008) fragt: „Was ist schiefgegangen?“

- Wie lässt sich ein solches Tatvorhaben schon im Vorfeld erkennen?

- Was kann man präventiv dagegen tun?

Der Schul-Amoklauf, der im März 2009 vom 17-jährigen Tim K. in der Albertville-Realschule von Winnenden bei Stuttgart verübt wurde und bei dem er 15 Personen tötete, rief in der Öffentlichkeit besonders großes Aufsehen und starkes Mitgefühl mit den Opfern und ihren Angehörigen hervor. Ähnlich wie 
nach dem Massaker an der Columbine-High-School in Littleton/USA gibt es für die deutsche Bevölkerung in der Wahrnehmung dieser Tat so etwas wie eine zeitliche Zäsur, also eine Zeit vor und nach dem Ereignis von Winnenden.

\subsection{Was ergaben die Untersuchungen?}

Der Abschlussbericht der Staatsanwaltschaft Stuttgart führt mehrere Details auf, angefangen von der Menge der gefundenen Patronenhülsen bis zur Anzahl der Vernehmungen und der beteiligten Polizisten, doch ein erkennbares Tatmotiv konnte nicht abgeleitet werden. Es wird aber das Bild eines jungen Mannes (Tim K.) gezeichnet, der kaum Freunde hatte, der von Mitschülern als zurückhaltend, still, nicht sehr zugänglich, aber durchaus freundlich beschrieben wird. Er soll ein überdurchschnittlicher Tischtennisspieler, aber ein eher unterdurchschnittlicher Schüler gewesen sein. Er war Ziel von Hänseleien, die aber in ihrer Intensität und Wortwahl als ,jugendtypisch“ bezeichnet werden. Man sieht Hinweise für eine Angststörung, denn Tim K. suchte fünfmal ein kinder- und jugendpsychiatrisches Krankenhaus auf.

Er soll in den ambulanten Gesprächen seinen „Hass auf Mitmenschen“ geäußert haben. Seine Freizeit verbrachte er am liebsten am Computer. Die Analyse seines Computers ergab Hinweise, dass er im Internet zu den Amokläufen an der Columbine-High-School und in Erfurt recherchiert hatte. Zwei Tage vor der Tat habe er noch ein Ego-Shooter-Spiel an seinem PC gespielt. Eine Ankündigung der Tat wurde jedoch nicht gefunden.

Aus der Akte der Staatsanwaltschaft geht hervor, dass es Tim selbst klar gewesen sein muss, dass „mit ihm etwas nicht stimmte“. Bereits im November 2006 hatte er in eine Suchmaschine die Wörter „psychisch krank“ eingetippt. Im Frühjahr 2008 vertraute er sich seiner Mutter an, weshalb er dann in einer Klinik für Kinder- und Jugendpsychiatrie ambulant behandelt wurde. Seine Therapeuten scheinen aber offensichtlich die Gefahr unterschätzt zu haben. Die Eltern haben die Andersartigkeit ihres Sohnes verdrängt und die Lehrer nichts von seinen Problemen bemerkt. Weiter geht aus der Akte der Staatsanwaltschaft hervor:

- Tim ist ein stiller und scheuer Schüler gewesen. Warnsignale sind ignoriert worden.

- Man hat aus Hilflosigkeit über den „verstummten“ Jungen hinweggeschaut.

\section{Was ging in Tim K., dem Täter von Winnenden, wirklich vor?}

Im Abschlussbericht des Expertengremiums „Amok Baden-Württemberg (2009)“ wird festgestellt, dass Erzieher, Ausbilder, Schulpsychologen, Lehrer und Sozialarbeiter eine differenziertere Wahrnehmung von zunächst noch nicht gewalttätig wirkenden, eher durch sozialen Rückzug auffallenden Jugendlichen 
haben sollten. Aus Sicht der psychiatrischen Experten manifestieren sich Früherkrankungen der psychiatrischen Krankheitsbilder Depression, Schizophrenie und schizoaffektive Psychosen erstmalig in der Adoleszenzphase. Ängste im sozialen Umgang können sich dann zu einer "sozialen Phobie“ ausweiten.

\subsection{Unterschiedliche Erklärungsversuche}

Über die Frage nach den Ursachen des Schul-Amoklaufs von Winnenden und beim Versuch, Persönlichkeit und Motive von Tim K. zu rekonstruieren, kam es zu einem Streit der Gutachter mit völlig unterschiedlichen Ergebnissen. Während der Gutachter der Staatsanwaltschaft du Bois dem Täter „masochistische Obsessionen“ bescheinigt und diese auch für den Auslöser des Amoklaufs hält, meint der von den Eltern der Opfer bestellte Psychiater Winckler aus Tübingen, dass es „keinerlei Beweis dafür“" gebe (Eißeler 2009).

Während du Bois glaubt, Tim K. habe gezielt auf Frauen und Mädchen geschossen, ist Winckler vom Gegenteil überzeugt. Er betonte die Außenseiterrolle von Tim, und die überhöhten Leistungsansprüche, denen der 17-Jährige ausgesetzt gewesen sei. Tim K. sei ein „unreifer, zwischen Selbstzweifeln und Allmachtsfantasien schwankender Jugendlicher “ gewesen, der keine Freunde gehabt und sich vor allem über den Leistungssport definiert habe, darin stimmen die Gutachter überein. Der einzige Hinweis auf eine „masochistische Persönlichkeit“ aber seien Bondage-Bilder gewesen, die man auf Tims Computer gefunden habe. Sie hätten „verschnürte Männer“ gezeigt, die von Frauen gequält wurden. „Über die sexuellen Fantasien von Tim K. sei ansonsten „,faktisch nichts bekannt“, weder eigene Tagebuchaufzeichnungen noch Zeugenaussagen." (Eißeler 2009)

Als eine mögliche Ursache für den „Masochismus“ käme die „mächtige, frustrierende, aber unverzichtbare Mutter “infrage, was jedoch bisher nicht verifiziert werden konnte. Tims Mutter sei, als ihr Sohn etwa elf Jahre alt war, wegen einer Krebserkrankung längere Zeit stationär behandelt worden und für diese Zeit für Tim emotional nicht verfügbar gewesen. Andererseits schloss Winckler nicht aus, dass Tim unter dem Einfluss einer beginnenden Psychose gehandelt haben könne.

Das eigentliche Motiv von Tim K. konnte bis heute nicht geklärt werden. Bis auf einen kurzen widersprüchlichen Kommentar hinterließ er keine Botschaft, die das Motiv für seinen Amoklauf erhellen könnte. Die Anwendung der Methode einer „psychologischen Autopsie“ (Bannenberg 2010a) ist unter Experten umstritten.

Folgende zentrale Fragen blieben bisher unbeantwortet:

- Hat Tim K. gezielt weibliche Opfer ausgesucht oder waren sie eher Zufallsopfer?

- Stand er unter einem zu hohen Erwartungsdruck seines Vaters? 
- Hat möglicherweise das innere Dilemma, stets der Beste sein zu wollen und zugleich die Angst zu versagen, für eine explosive Mischung gesorgt?

- Was hatte sich Tim K. von seiner Selbstvernichtung versprochen?

Waldrich (2007) vermutet, Tims Vater sei es wichtig gewesen, dass sein Sohn zu den „Siegern“ zählte. Wenige Tage vor dem Massaker hatte Tim offenbar beim Tischtennis haushoch verloren. Vielleicht war dies der letzte „Funke“, der in ihm die Katastrophe auslöste. Unter dem Druck von Beschämung und der daraus resultierenden Aggression habe er während der Tat den Unterschied zwischen der Alltagswirklichkeit und seinen Gewaltfantasien nicht mehr erkennen können. Von der Polizei in die Enge getrieben, sei er aufgrund seiner „kurzzeitigen Schizophrenie“ am Ende wohl zusammengebrochen. Das sei der Anlass für ihn gewesen, sich zu erschießen.

Heitmeyer (2009) sieht in den Handlungen von Tim K. eine Verquickung persönlicher und sozialer Faktoren. Tim K. habe sich in einem für ihn unlösbaren Konflikt befunden. Er sollte stark und erfolgreich sein, habe aber dafür nicht das notwendige seelische Rüstzeug besessen. Wie viele andere Jugendliche habe er seine Überlegenheitsfantasien gepflegt, ohne die Möglichkeit zu haben, sie in einer Weise umzusetzen, für die er hätte Anerkennung bekommen können. Für Heitmeyer (2009) sind Schul-Amokläufer keine kranken Außenseiter. Er nimmt an, dass sie ihre Erfolgs- und Überlegenheitsideologie mit vielen anderen Jugendlichen teilen.

Obleser (2010) versuchte, die seelische Verfassung von Tim K. anhand der ihm bekannten Fakten zu rekonstruieren. Für ihn sind seine Handlungsweisen auch ohne psychiatrische Diagnose nachvollziehbar. Tim K. habe in erster Linie unter hartem Erfolgsdruck und gleichzeitig unter einem dramatischen Mangel an Liebe und Zuwendung gelitten. In der Schule sei er nicht gut vorangekommen. „Star“ der Familie sei seine jüngere Schwester gewesen, die erfolgreicher und beliebter gewesen sei als der ältere Tim. Der Vater, ein Unternehmer, habe von seinem Sohn vor allem Durchsetzungskraft und Leistung erwartet, das auch im Tischtennis- und im Schützenverein. Wenn Tim Erfolge vorweisen konnte, sei er gelobt und mit Geld belohnt worden. War er jedoch gescheitert, sei er vom Vater mit Nichtachtung gestraft worden. Außerdem sei er bei Mädchen nicht gut angekommen.

Der daraus resultierende Hass sei das Ergebnis einer „nach Liebe und Anerkennung hungernden Seele“. In der Nacht vor dem Amoklauf hatte sich Tim K. etwa 120 Bondage-Bilder, auf denen gefesselte Männer von Frauen gequält werden, aus dem Internet auf seinen Rechner geladen. Während der Gutachter du Bois (2010) diesen Umstand als klaren Hinweis auf eine sexuelle Perversion interpretiert, sieht Obleser (2010) in den Bildern eher einen „symbolischen Ausdruck für die Qual der gefesselten Seele“. Tim K. habe sich „nach Nähe und Liebe gesehnt, sie jedoch nicht erhalten“. 
Auch um die Bedeutung und Wirkung der Gewaltmedien gibt es unterschiedliche Meinungen. So hält Lempp (2009) die prägende Kraft von Bildmedien auf die Köpfe von Jugendlichen für nahezu ,verheerend“. Insbesondere in der Fantasie potenzieller Amokläufer sollen sich unter dem Eindruck von Gewaltbildern „Nebenrealitäten“ aufbauen. Diese Parallelwelten könnten eine Dimension erreichen, die das echte Leben zu verschütten drohe. Löst eine Kränkung oder Blamage, etwa in der Schule, dann intensive Gefühle wie Wut oder Scham aus, könne der letzte Bezug zur Wirklichkeit zusammenbrechen und der Täter „psychotisch“ reagieren.

Lempp (2009) spricht von einer „Zehn-Minuten-Schizophrenie“ - das sei ungefähr die Zeitspanne, die das Massaker dauert. Täter wie Tim K. sind nach Lempp (2009) nicht geisteskrank, aber vorübergehend würden sie es, sobald der Entschluss zum Amoklauf gefasst sei. Lempp verweist auch darauf, dass Störung im Wechselspiel mit dem kulturellen und sozialen Milieu, in dem die Täter aufwachsen, entstehen, worauf sie schließlich so brutal reagieren. Lempps Auffassung verschiebt den Schwerpunkt der Problematik dorthin, wo es für Menschen, die zu einer einseitigen Betrachtung neigen, unbequem wird.

\section{Die Frage lautet nicht mehr: „Wie krank ist der Täter?“, sondern:}

\section{„Wer ist hier überhaupt krank, der Täter, seine Umwelt oder beide?“}

Die widersprüchlichen Auffassungen und Erklärungsversuche zwischen den Experten sind insofern mehr als ein Gutachterstreit, denn die Frage nach den Ursachen für die entsetzliche Tat ist auch eine Frage nach der öffentlichen Schuld: „Hätte der Amoklauf verhindert werden können? Wenn ja, wie?“

Wie häufig nach dramatischen Anlässen wird als erstes nach Verschärfung von Gesetzen gerufen. Im Falle von Winnenden nach der Verschärfung des Waffengesetzes, weil der Vater Mitglied in einem Schützenverein war, Tim einige Male wohl selbst mitkommen konnte und der Vater die von Tim benutzte Pistole nicht ordnungsgemäß verschlossen hatte. Schützenvereine mit ihren 1,5 Millionen Mitgliedern, organisiert in 15.0oo Vereinen, gelten in Deutschland jedoch als gewachsener Teil der Heimatkultur. Die Sportschützen befürchteten nach dem Ereignis von Winnenden eine Kriminalisierung ihrer Sportart. Schulen sollten nun „ballerfreieZonen“ werden. Schießstände in Schulen sollten an Schützenvereine nicht mehr vermietet werden. In NRW gab es damals 16o Schießkeller an den Schulen. Grafe (2010) forderte in der Süddt. Zeitung verstärkte Kontrollen der sicheren Aufbewahrung von Waffen und Munition in Räumlichkeiten der Waffenbesitzer. Aufgrund der staatlichen Schutzpflicht müsse der Staat dort, wo er Risikobereiche nicht ausreichend absichern könne, Verbote aussprechen, insbesondere dann, wenn die drohende Gefährdungslage unbeherrschbar sei. Er kann sich auf ein For- 
schungsergebnis der Kriminologin Bannenberg (2010a) stützen, wonach die Verfügbarkeit von Schusswaffen der höchste Risikofaktor für eine Tatausführung sei.

Im Juni 2009 wurde vom Bundestag schließlich eine Gesetzesnovellierung zum Waffengesetz beschlossen, die in der Praxis nach Meinung von Experten wohl wenig verändern wird. Die Lobbyarbeit von zwei Millionen Schützen scheint offensichtlich gewirkt zu haben.

Der Vorsitzende eines Sonderausschusses des Landtags von Baden-Württemberg, der CDU-Abgeordnete Palm (2009), beschrieb auf einer Tagung in der Evangelischen Akademie in Bad Boll am 30.9.2009 fünfwichtige Handlungsfelder:

1. Früherkennung

2. Stärkung des Erziehungsauftrags der Eltern

3. Medienkompetenz

4. Sicherheit an Schulen

5. Waffenrecht.

Konkrete Maßnahmen für die Erhöhung der Sicherheit von Lehrern und Schülern wurden nach dem Ereignis von Winnenden in einigen Schulen durchgeführt, wie einheitliche Nummerierungen der Schulräume. Die bisherigen Amokereignisse hatten gezeigt, dass bei den Notrufen die Retter zwar gewusst hätten, die Klasse X habe sich verbarrikadiert, aber wo genau sie sich im Schulgebäude befand, sei ein Rätsel geblieben.

In jedem Klassenzimmer sollen nun kleine Tafeln mit den nötigen Verhaltensregeln für den Notfall platziert werden. Spezielle Alarmsignale, die über die teils schon vorhandenen oder ansonsten noch einzubauenden Lautsprecherund Klingelanlagen verbreitet werden, sollen eingerichtet werden. Die Übersicht der Flucht- und Rettungswege soll aktualisiert werden. Sämtliches Material soll digitalisiert und kreisweit zentral gespeichert werden. Wenn Feuerwehr oder Polizei einen Einsatz in der Schule haben, sollen sie zukünftig nicht mehr in einem Stapel Karten wühlen müssen, sondern sich digital den nötigen Überblick verschaffen können.

Weil ein vernetztes Vorgehen am meisten Erfolg verspricht, ist die Kooperation zwischen Schulbehörden, Schulpsychologen, Jugendhilfe und Kinder- und Jugendpsychiatrie, Polizei, Gerichten und Presse auf lokaler Ebene zu verstärken. Es ist ein Kommunikationssystem zu erarbeiten, das den Schulen ermöglicht, die anderen Stellen über kritische Vorfälle, akute Bedrohungen oder über einen Verdacht schnell zu berichten.

In jeder Schule ist ein Krisenteam zu implementieren, das die folgenden Aufgaben übernimmt: 


\section{Wie ist die Interdisziplinäre Kooperation einzurichten?}

- Warnsignale, Drohungen und daraus resultierende Gefahren sind zutreffend einzuschätzen.

- Vorgeschichte, Verhalten und Kontakte eines potenziellen Täters sind zu analysieren.

- Wechselwirkungen zwischen Täter, Vorgeschichte, Situation und Zielpersonen sind festzustellen.

- Zwischen wagen Drohungen und echten Gefahren ist zu unterscheiden.

- Es ist festzustellen, ob es sich um Nachahmer oder Trittbrettfahrer handelt.

- Mit der Presse sind Vereinbarungen zu treffen, ausgewogen zu berichten.

- Bei Gerichten ist auf eine möglichst schnelle Verurteilung der Täter zu drängen, um die Zahl der Trittbrettfahrer einzudämmen.

- Eine Überbewertung von Warnsignalen und eine Stigmatisierungen verdächtiger Schüler ist zu vermeiden.

- Die Entwicklung von Hysterien und einer "destruktiven Gerüchteküche“ muss verhindert werden.

In der Zwischenzeit wurden ausführliche Maßnahmen- und Fragekataloge entwickelt, z.B. im Bundesland Hessen, in denen Schritt für Schritt die Vorgehensweise von Lehrern, Eltern und Mitschülern nach einer Ankündigung einer bedrohlichen Äußerung oder bei Verhaltensauffälligkeiten eines Schülers dargelegt wird (Bannenberg, 2010a).

\section{Für ein Bedrohungsmanagement müssen Leitsätze entwickelt werden.}

Eine besonders klare Beschreibung des Täterprofils und des Tatvorgangs konnten von Matti Saari, dem Schul-Amokläufer von Kauhajoi (Finnland) erstellt werden.

„Matti Saari stammt aus einem kleinen finnischen Ort mit rund 6.000 Einwohnern sowie hohen Arbeitslosen- und Selbstmordquoten. Viele ältere Einwohner sind extrem religiös und hängen Erweckungsbewegungen an; Fernsehen oder Theater sind für sie Teufelszeug.

Matti Saari wuchs in schwierigen Verhältnissen auf. Seine Eltern trennten sich, als er noch keine sieben Jahre alt war. Saari war ein stiller Junge, der in der Mittelschule gemobbt wurde. ,Niemand wollte sein Freund sein, alle haben ihn ignoriert und empfanden ihn als unangenehmen Typen', sagt eine Mitschülerin von damals, ,auch vom Wehrdienst wurde er vorzeitig suspendiert‘.

Der Junge habe sich immer mehr abgekapselt, sagt Schuldirektor Varmola. Ähnlich seinem Vorbild Pekka-Eric Auvinen trug er mit etwa 16 Jahren vor allem weite, dunkle Kleidung. Immer tiefer verschwand er in der Welt des Internets mit Schießspielen und Foren, auf denen sich Menschen gegenseitig in 
ihrem Hass bestätigten. Seine Mitschüler zogen ihn auf. Beide, Auvinen und Saari, sollen über einen längeren Zeitraum Antidepressiva bekommen haben.

Wie isoliert sich Matti Saari bis zum Schluss fühlte, zeigt eine Kontaktanzeige, die er noch im August bei einem Internetservice aufgab. Er sei 1,72 Meter groß, wolle keine Kinder haben, interessiere sich für Computer, Schlagzeug, Sex, Bier und Schießübungen mit seiner Walther, und er suche eine 16- bis 25-ährige Partnerin. Etwa zur selben Zeit erhielt er seinen Waffenschein. Damit fuhr er nach Jokela und kaufte seine Pistole im selben Geschäft, in dem auch PekkaEric Auvinen Kunde gewesen war.

Schon einige Zeit vor der Tat hatte die Polizei von Saaris militanten YouTubeAuftritten erfahren. Es gab sogar die Anweisung, dessen Waffe am Freitag vor der Tat zu konfiszieren. Doch selbst als der Verdächtige am Montag im Kommissariat erschien, passierte nichts. Seither war Matti Saari klar, dass inm die Polizei auf der Spur war. Sein lang geplanter Privatkrieg gegen die Menschheit konnte nicht mehr warten.

Am 24.09.2008, auf dem Weg zur Schule, wo sein Kurs eine Arbeit schreibt, zieht Saari plötzlich eine schwarze Maske über den Kopf. Es ist 10:40 Uhr. Er holt eine Pistole aus seiner Umhängetasche, eine Walther P22, reißt die Tür zum Klassenzimmer auf und feuert auf seine Mitschüler. Panik bricht aus. Drei von ihnen können durch ein Fenster fliehen. Die meisten werfen sich auf den Boden. Der Vermummte mit der schwarzen Lederjacke richtet seine Waffe auf die Wehrlosen, er exekutiert einen nach dem anderen. Zehn Menschen sterben bei dem Massaker, unter ihnen acht Schülerinnen, ein Kommilitone sowie der Dozent Kimmo Martiskainen, 51, der sich Matti mit allen Kräften entgegengeworfen hatte." (Stern Ausgabe 41/2008e) 